\title{
Anomalous Propagation Characteristics of Airy Beam in Nonlinear Kerr Media
}

\author{
Li Shao, Yun-Long $W u$ * and Qing Ye
}

State Key Laboratory of Pulsed Power Laser Technology, National University of Defense Technology,

Hefei 230037, China; shaoli17@nudt.edu.cn (L.S.); yeqing18@nudt.edu.cn (Q.Y.)

* Correspondence: wuyunlong17@nudt.edu.cn

\begin{abstract}
The propagation characteristics of a single Airy beam in nonlinear Kerr media were numerically investigated by utilizing the split-step Fourier transform method. We show that in addition to normal breathing solitons, the anomalous bound states of Airy spatial solitons can also be formed, which are similar to the states formed in the interaction between two Airy beams in nonlinear media. This quasi-equilibrium state is formed by the interaction of the main soliton beam and side lobes of Airy beam due to their different propagation trajectories in the nonlinear media. Moreover, it has been shown the Airy spatial solitons in tree structure can be formed by adjusting the initial parameters in the interaction between the Airy beam and Kerr media.
\end{abstract}

Keywords: Airy beam; propagation; nonlocal Kerr media; solitons

\section{Introduction}

In 1979, Berry and Balazs first demonstrated that there existed a special solution to the Schrödinger equation which was called "Airy packet" in the context of quantum mechanics [1]. The special wave packet had the unique and fantastic characteristics of non-diffraction, self-acceleration and self-healing. However, the achievement did not attract sufficient attention due to the fact that the ideal Airy packet carried infinite energy, which could not be realized in the experiments. In 2007, it was found that the Airy function truncated by exponentially decaying was also a particular solution to the Schrödinger equation [2], which helped to make the energy of the Airy packet become finite. Subsequently, fascinating Airy beams, which had the characteristics of non-diffraction, self-acceleration and self-healing, were first realized by utilizing a liquid crystal spatial light modulator in the experiments [3]. This study on the new Airy beams and other non-diffractive beams has become a hot area in recent years. Recently, most attention has been concentrated on the generation of Airy beams [4-8], particular Airy beams [9-13], the control of particular beams [14-16] and the potential application of these beams, such as the directional control of particles [17] and the generation of light bullets [18,19].

Recently, it has been shown that the stable spatial solitons can be formed when the Airy beam propagates in the nonlinear media and interacts with the media. Thus, more and more works are being concentrated on the propagation characteristics of the Airy beam in different kinds of nonlinear media [20-22]. The propagation and interactions of Airy beams in nonlinear media contain many novel dynamics. Bound state and unbound soliton pairs, as well as single solitons, can be formed during the interactions between in-phase and out-of-phase Airy beams in Kerr [23] and saturable [24] nonlinear media. The emerging soliton parameters depend on the initial characteristics of the Airy pulses [25]. Further increase in intensity can result in the creation of additional solitons [26]. The interaction between two finite Airy-Gaussian beams has been investigated in different media with the defected photonic lattices [27]. Bekenstein et al. investigated and exhibited the complex nonlinear dynamics of wave packets in an Airy beam [28]. It was also found that the in-phase and out-of-phase Airy beams can present attraction and repulsion under proper 
conditions of interval and initial launch angle [29]. In a nonlinear fiber, the interactions between a colliding Airy pulse and a temporal soliton pulse at the center frequency were also studied [30].

Nevertheless, the study on the anomalous propagation characteristics of a single Airy beam in nonlinear Kerr media has barely been reported. Therefore, we numerically demonstrated the corresponding study by utilizing the split-step Fourier method. The impacts of different initial parameters on the formation and evolution of Airy spatial solitons in the nonlinear Kerr media were also investigated through numerical simulation. We hope our work can provide some theoretical groundwork for future experimental study on the propagation characteristics control of Airy beams in nonlinear media.

\section{Theoretical Model}

The Schrödinger equation, which governs the propagation dynamics of 1D Airy beams, could be described as

$$
i \frac{\partial \phi}{\partial \xi}+\frac{1}{2} \frac{\partial^{2} \phi}{\partial s^{2}}+\frac{n_{2}}{n_{0}}|\phi|^{2} \phi=0
$$

where $\phi$ is the electric field envelope, $s=x / x_{0}$ represents a dimensionless transverse coordinate, $x_{0}$ is an arbitrary transverse scale, $\xi=z / k x_{0}^{2}$ represents the normalized propagation distance, $k=2 \pi n / \lambda_{0}$ is the wave number, $\lambda_{0}$ is the center wavelength, $n_{2}$ is the third-order refractive index, $n_{0}$ is the linear refractive index of media. Due to the fact it is hard to acquire the accurate solution for Equation (1), we utilize the split-step Fourier method to investigate the propagation characteristics of a single Airy beam in nonlinear Kerr media with the initial condition of a 1D finite Airy beam, which can be expressed as

$$
\phi(\xi=0, s)=A i(s) \exp (a s)
$$

where $a$ is the decay factor, and the $A i(\cdot)$ represents the Airy function [31].

Here, we add two extra parameters to Equation (2) to adjust the amplitude distribution of the initial light field, which would make it easier to get an insight on the impacts of initial parameters to the propagation properties of an Airy beam in Kerr media. The modified initial condition can be expressed as

$$
\phi(\xi=0, s)=A \cdot A i\left(\frac{s}{\chi_{0}}\right) \exp \left(\frac{a s}{\chi_{0}}\right)
$$

where the $\chi_{0}$ is the amplitude distribution factor and $A$ is the initial maximum amplitude of the incident beam.

\section{Numerical Simulation and Results Analysis}

\subsection{Impacts of Airy Beam with Different Initial Amplitudes on Propagation Characteristics in} Kerr Media

Firstly, we come to the propagation characteristics of a 1D Airy beam in Kerr media along the $x-z$ plane under the condition of $x_{0}=130 \mu \mathrm{m} ; \lambda_{0}=632.8 \mathrm{~nm} ; \chi_{0}=1 ; a=0.15$; $n_{2}=10^{-5} \mathrm{~m}^{2} \mathrm{~W}^{-1}$; and $n_{0}=1.45$, which are shown in Figure $1 \mathrm{a}-\mathrm{i}$.

It can be seen from Figure 1a that when the initial amplitude of the Airy beam is small, its propagation characteristics in Kerr media are barely affected by the nonlinear effect, which can be ignored. By increasing the initial amplitude of the Airy beam, as shown in Figure 1b, compared with Figure 1a, it can be found that the main lobe propagation of Airy beam is basically unaffected, but part of its side lobe energy begins to be self-trapped and forms new light radiation. The new beam will no longer follow the parabolic path of the Airy beam but will travel along a straight line. By continuing to increase the initial amplitude of the Airy beam, as shown in Figure 1c, the diffraction effect and the nonlinear Kerr effect of an incident beam propagating in the media reach a preliminary balance, so the generation of breathing optical soliton and the formed spatial soliton propagating along the path of straight line can be observed. When the initial amplitude increases further, as shown 
in Figure 1d,e, the equilibrium effect is further strengthened, which shows that the formed soliton state is more stable. In order to facilitate the comparison of the different soliton states shown in Figure 1d,e, the curves of the maximum light intensity of Figure 1d,e with the propagation distance can be made, respectively, as shown in Figure 1i. It can be seen from Figure $1 \mathrm{i}$ that the periodic soliton state corresponding to Figure $1 \mathrm{~d}$ is formed and maintained in the later propagation process, while the periodic soliton state corresponding to Figure 1e is formed after a short propagation distance and can exist stably in quite a long propagation distance. Therefore, it can be considered that in a certain range, the larger $A$ is, the easier the formation of a spatial soliton state is.

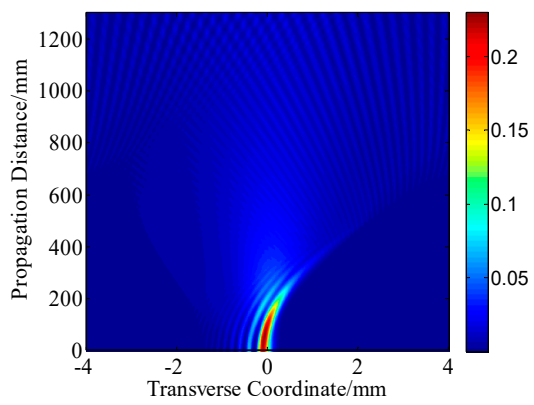

(a)

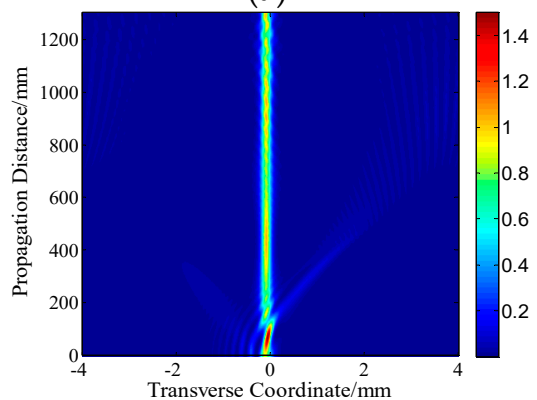

(c)

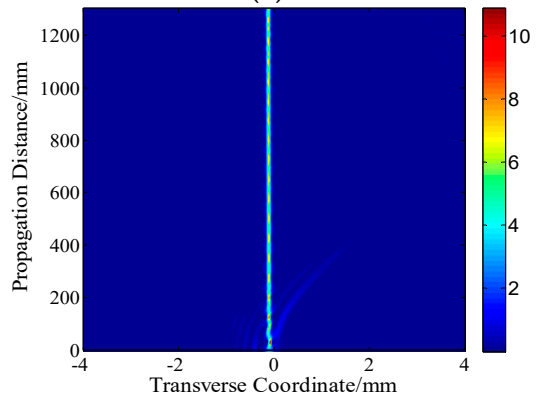

(e)

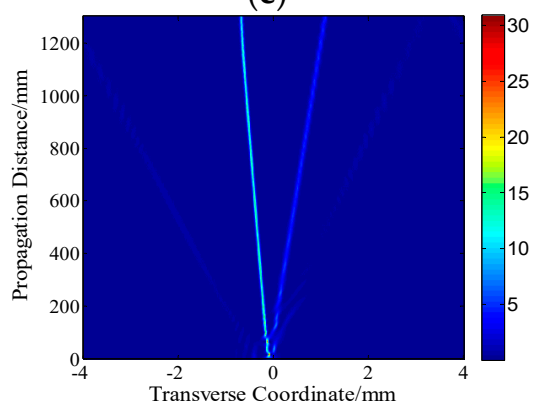

(g)

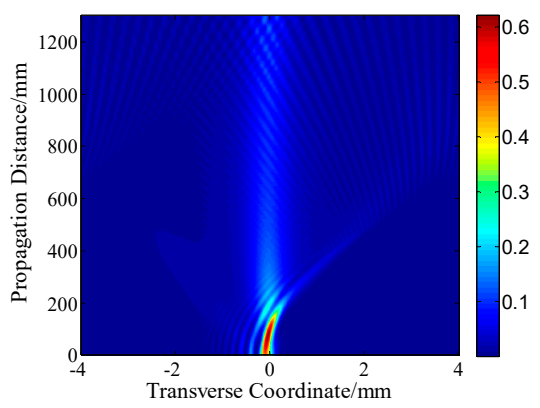

(b)

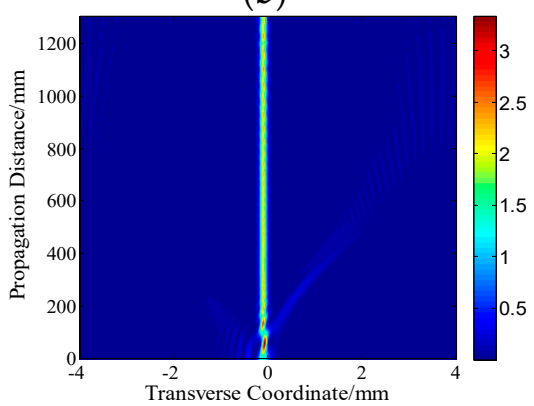

(d)

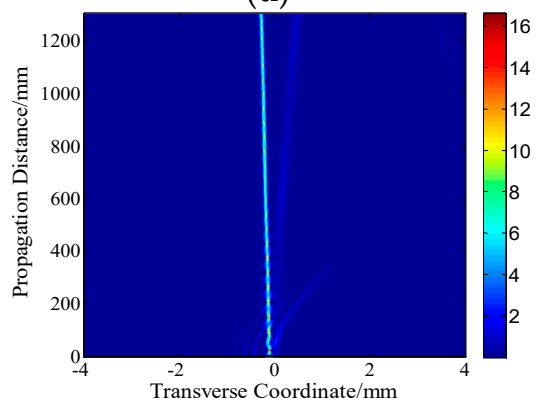

(f)

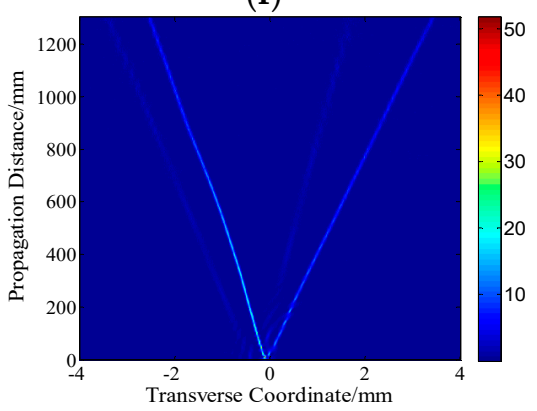

(h)

Figure 1. Cont. 


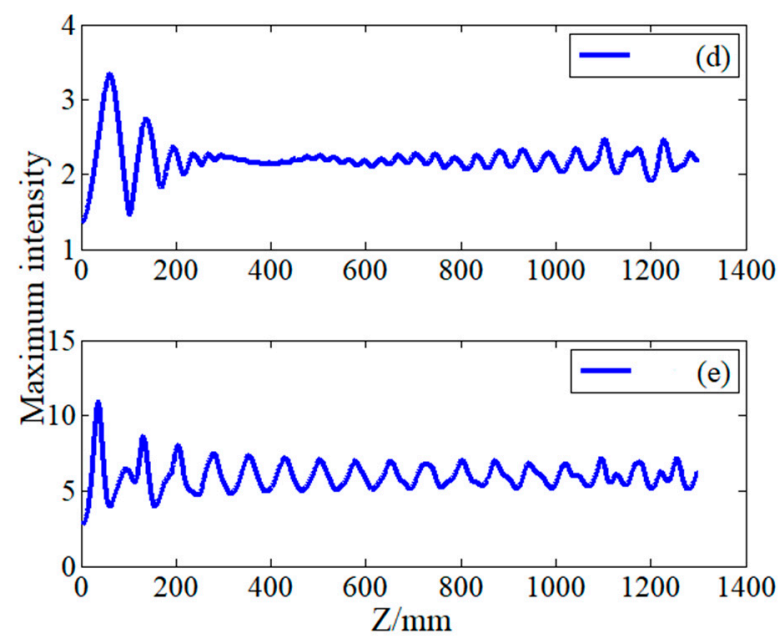

(i)

Figure 1. The propagation property of a 1D Airy beam in Kerr media with different initial amplitudes: (a) $A=1 ;$ (b) $A=1.5 ;$ (c) $A=2$; (d) $A=2.5$; (e) $A=3.5$; (f) $A=4 ;$ (g) $A=5$; (h) $A=6$; and (i) is the maximum intensities of the beam in $(\mathbf{d}, \mathbf{e})$ versus propagation distance.

When the initial amplitude of the Airy beam increases further, as shown in Figure 1f, it can be found that the aforementioned equilibrium state begins to break. Under this condition, although there are also relatively stable breathing optical solitons, it can be clearly seen that there is a divergence between the energy of the main lobe and the side lobe. Part of the side lobe is separated from the soliton beam and forms a new beam to propagate in the media. At this time, the energy of the main beam is weakened. It can also be seen from Figure $1 \mathrm{f}$ that due to the breaking of the balance effect, the beams formed by the main lobe and the side lobe have different velocity directions, respectively, so that the main beam no longer propagates in the vertical direction but propagates in a certain angle along the oblique direction. When the initial amplitude of the Airy beam continues to increase, as shown in Figure $1 \mathrm{~g}$, $\mathrm{h}$, the balance effect of soliton state is further broken, and the divergence effect is continuously strengthened, which eventually leads to the weakening of the main beam. More and more beams are separated and propagate independently, and the angle between the propagation tracks of each beam is continuously increased.

\subsection{Impacts of Kerr Media with Different Third-Order Refractive Indexes on the Propagation Characteristics of Airy Beam}

In this section, we mainly focused on the impacts of Kerr media with different thirdorder refractive indexes on the propagation properties of single Airy beam under the initial condition of $x_{0}=130 \mu \mathrm{m} ; \lambda_{0}=632.8 \mathrm{~nm} ; \chi_{0}=1, a=0.15$; and $A=1.6, n_{0}=1.45$; which are shown in Figure $2 \mathrm{a}-\mathrm{f}$.

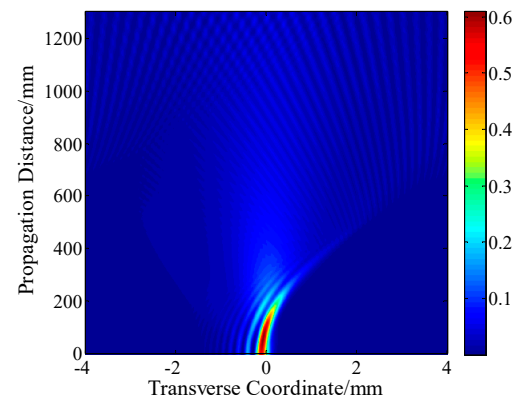

(a)

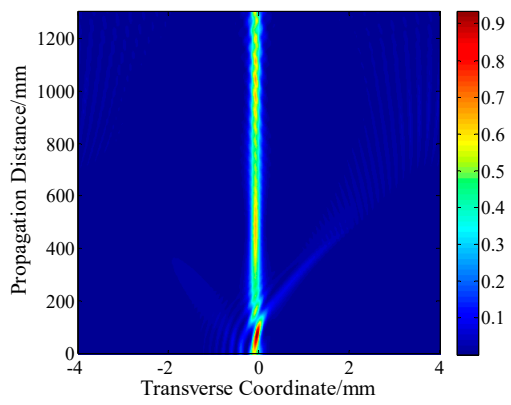

(b)

Figure 2. Cont. 


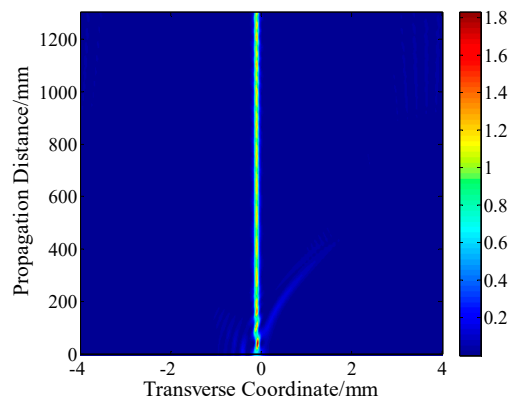

(c)

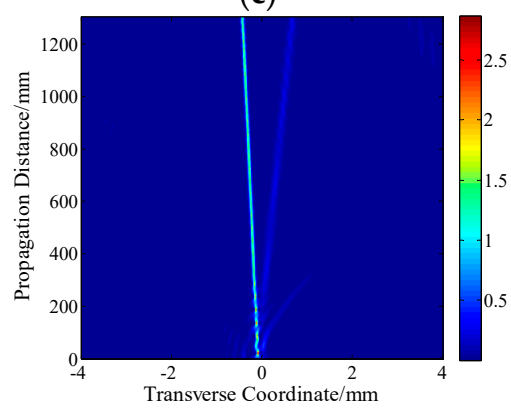

(e)

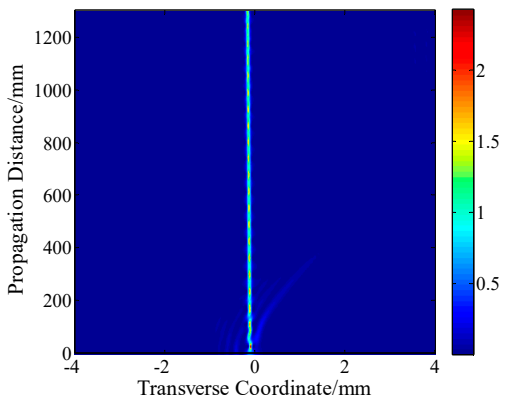

(d)

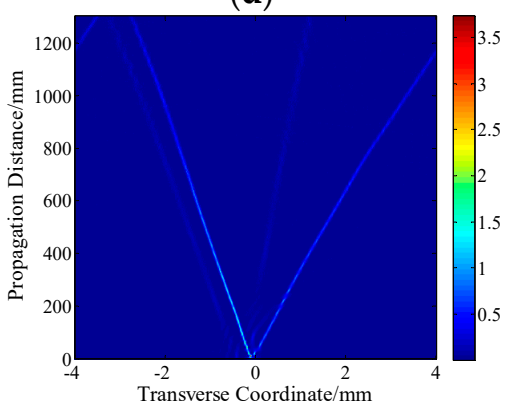

(f)
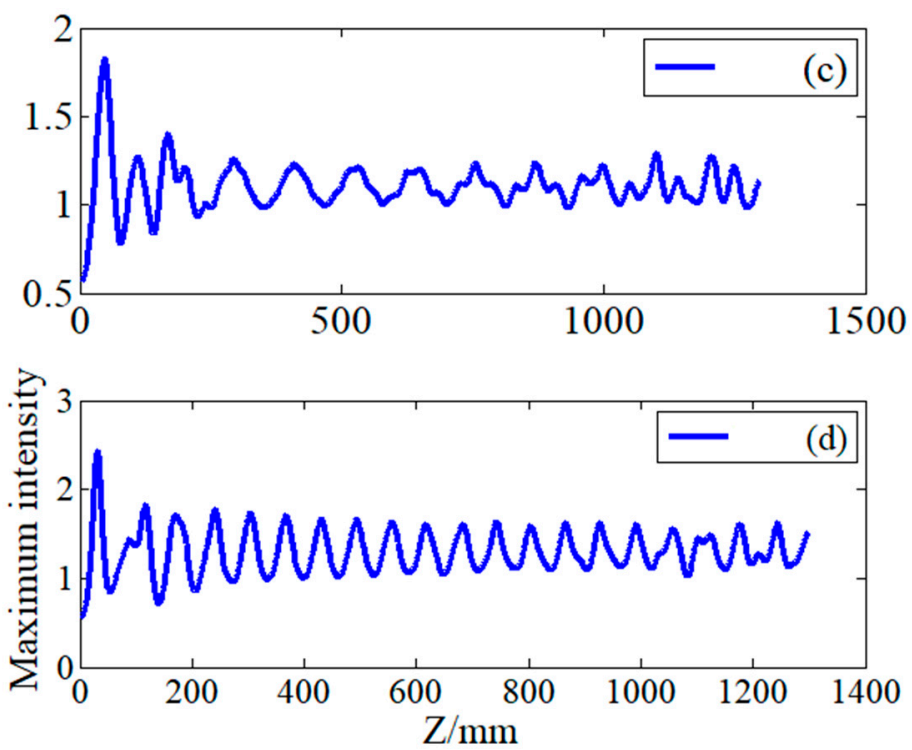

(g)

Figure 2. The propagation property of a 1D Airy beam in Kerr media with different third-order refractive indexes: (a) $n_{2}=5 \times 10^{-6} \mathrm{~m}^{2} \mathrm{~W}^{-1}$; (b) $n_{2}=1.5 \times 10^{-5} \mathrm{~m}^{2} \mathrm{~W}^{-1}$; (c) $n_{2}=3.5 \times 10^{-5} \mathrm{~m}^{2} \mathrm{~W}^{-1}$; (d) $5.5 \times 10^{-5} \mathrm{~m}^{2} \mathrm{~W}^{-1}$; (e) $7.5 \times 10^{-5} \mathrm{~m}^{2} \mathrm{~W}^{-1}$; (f) $15 \times 10^{-5} \mathrm{~m}^{2} \mathrm{~W}^{-1}$; and (g) is the maximum intensities of the beam in $(\mathbf{c}, \mathbf{d})$ versus propagation distance.

It can be seen from Figure 2 that when the initial amplitude of the Airy beam is constant and only the nonlinear refractive index of Kerr media is changed, the propagation characteristics of the Airy beam in Kerr media is similar to Figure 1. Therefore, it can be considered that the effect of parameter $n_{2}$ on the propagation characteristics of the Airy beam in the media is similar to that of $A$. However, it should be noted that the equilibrium effect required for the soliton state is more sensitive to the change of initial amplitude than the change of nonlinear refractive index. For example, to achieve the state shown in Figure 1e, the incident amplitude needs to be increased by 3.5 times the initial condition, 
while to achieve a similar state, as shown in Figure 2d, the nonlinear refractive index needs to be increased by 11 times the initial condition.

3.3. Impacts of Different Distribution Factors on the Propagation Characteristics of Airy Beam in Kerr Media

In this section, we will investigate the impacts of different distribution factors on the propagation characteristics of a single Airy beam in Kerr media under the initial condition of $x_{0}=130 \mu \mathrm{m}, \lambda_{0}=632.8 \mathrm{~nm}, n_{2}=1.2 \times 10^{-5} \mathrm{~m}^{2} \mathrm{~W}^{-1}, a=0.15, A=1.6, n_{0}=1.45$, which are shown in Figure 3(a1-f1).

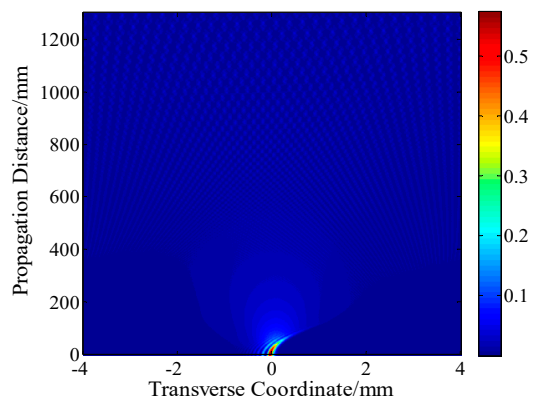

(a1)

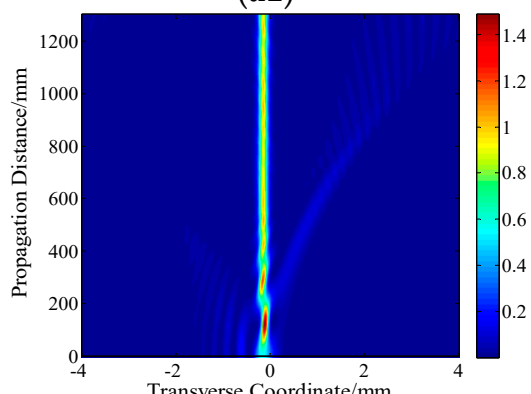

(b1)

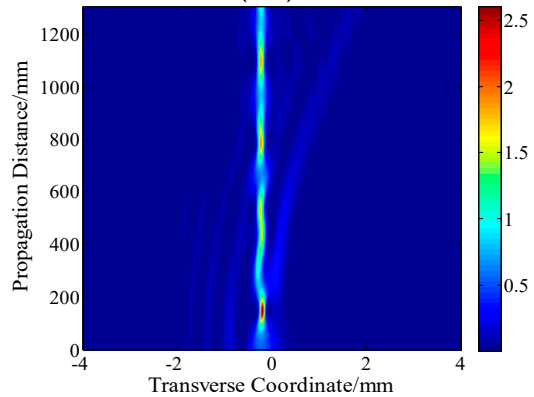

(c1)

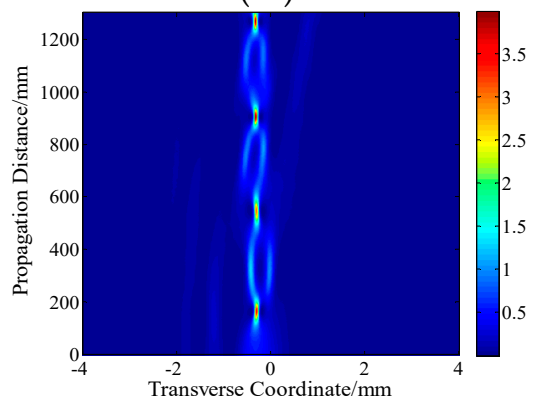

(d1)

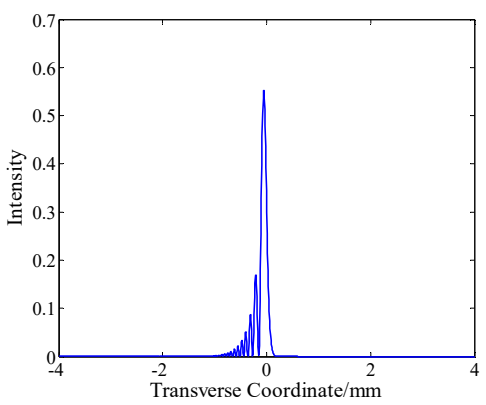

(a2)

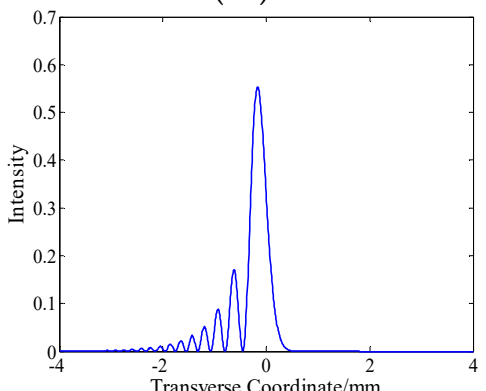

(b2)

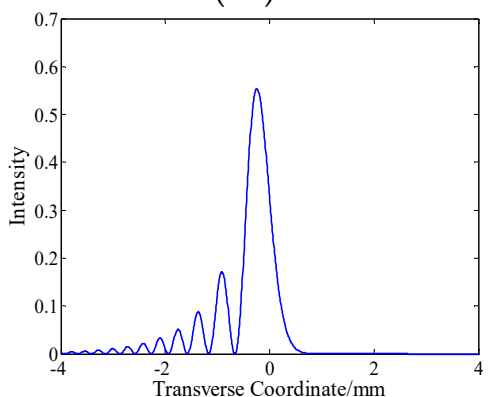

(c2)

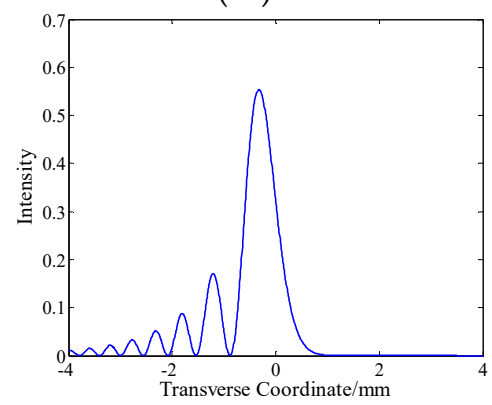

(d2)

Figure 3. Cont. 


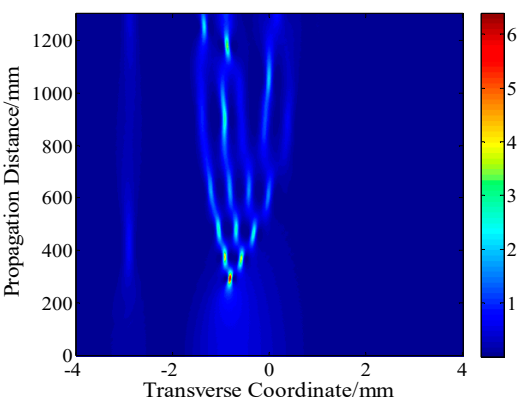

(e1)

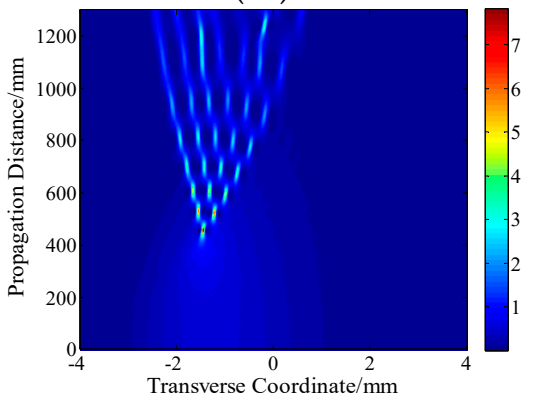

(f1)

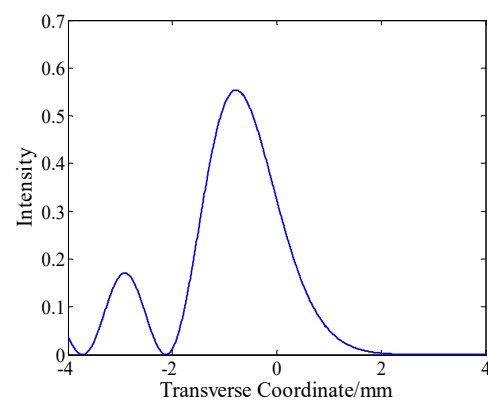

(e2)

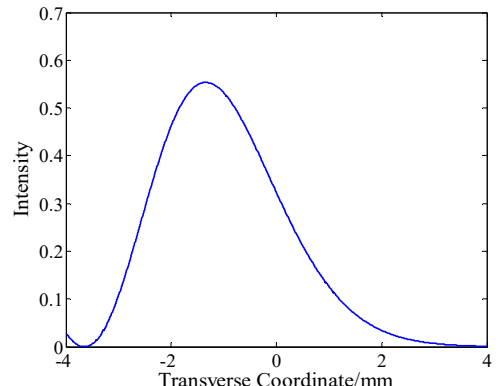

(f2)

Figure 3. The propagation property of a 1D Airy beam in Kerr media with different distribution factors: (a1) $\chi_{0}=0.5 ;(\mathbf{b} 1) \chi_{0}=1.5 ;(\mathbf{c} 1) \chi_{0}=2.2 ;(\mathbf{d} 1) \chi_{0}=2.9 ;(\mathbf{e} 1) \chi_{0}=7 ;(\mathbf{f 1}) \chi_{0}=12 ;$ and (a2-f2) are the corresponding initial intensity distributions for (a1-f1).

It can be seen from Figure 3 that when the incident Airy beam has different distribution factors, its propagation in Kerr media will show some very different anomalous characteristics in Figures 1 and 2. When the distribution factor is small, as shown in Figure 3(a1,a2), the nonlinear nonlocal effect has little effect on the propagation characteristics of an Airy beam. When the distribution factor increases, as shown in Figure 3(b1,b2), it can be seen from the comparison between Figure $3(\mathrm{a} 2, \mathrm{~b} 2)$ that the full width at half maximum of the main lobe and the side lobe of the incident beam are increased. It can be considered that the energy distribution curve of the Airy beam is stretched in the transverse direction as a whole, due to the modulation of the distribution factor. When the modulated Airy beam propagates in Kerr media, the nonlinear self-focusing effect and the linear diffraction effect reach a balance, and the breathing optical soliton is formed. If the distribution factor continues to increase, as shown in Figure 3(c1,c2), the balance effect established in the previous state is broken, and the change in the energy distribution of the incident beam makes the energy of some side lobes no longer participate in the construction of the soliton state, but still keeps the singularity of the Airy beam propagating independently in the media. In addition, an interesting phenomenon can also be observed, i.e., because the newly constructed main soliton beam propagates in the media in the path of an approximate straight line, and the independent sidelobe propagates along the path of parabola, so the two tracks overlap in space, which leads to the interaction between the main beam and the sidelobe in the media. As a result, the transverse self-acceleration motion of the sidelobe is basically cut off, and the propagation path of the main beam is partially cut off at the positions of the interaction, resulting in the obvious weakening of the light intensity at these positions. When the distribution factor is further increased, as shown in Figure $3(\mathrm{~d} 1, \mathrm{~d} 2)$, the interaction between the main beam and the side lobe in the media is further enhanced, resulting in the establishment of a new quasi equilibrium state. Interestingly, the optical soliton formed in this equilibrium state is significantly different from the breathing soliton state shown in Figure 3(b1). The soliton state shown in Figure 3(d1) is a kind of bound state of spatial solitons, which is very similar to the bound state formed by the interaction between two Airy beams in the media. Meanwhile, because the side 
lobes and the main soliton beam have different spatial structures and propagate along different trajectories in the media, the soliton bound states formed during their interaction are not completely symmetrical in space. However, the new soliton state can still propagate in a diffraction-free state within a certain distance. If the distribution factor continues to increase, as shown in Figure 3(e2), only one main lobe and one side lobe remain in the transverse range of the intensity distribution curve. It can be seen from Figure 3(e1) that the side lobe is hardly involved in the interaction with the main lobe. At this time, the optical solitons formed by the interaction between the main lobe and the media will propagate in a special kind of spatial structure which is similar to a "tree". Specifically, when the main lobe propagates in the media for a certain distance, it will reach the balance of a linear effect and nonlinear effect due to the interaction with the media, resulting in the formation of a single spatial optical soliton. With the increasing propagation distance, one optical soliton will be divided into two solitons for independent propagation, and then it will be divided into three, increasing in sequence. After each differentiation, the energy of the new optical soliton will be weakened. Therefore, this special propagation state will not be maintained for a long distance. After a long-distance propagation, it will change from orderly propagation to disordered propagation. When the distribution factor is further increased, as shown in Figure 3(f2), only one main lobe remains in the transverse range of the intensity distribution curve of the incident beam. At this time, the main lobe carries most of the energy of the incident beam and propagates independently in the Kerr media. From Figure 3(f1), it can be seen that the optical soliton formed under this condition still propagates in a "tree" spatial structure, and the propagation distance to keep this special propagation state becomes longer.

\section{Conclusions}

We numerically demonstrated the anomalous propagation characteristics of a single Airy beam in nonlinear Kerr media by utilizing the split-step Fourier transform method. It was found that the effect of third-order refractive index on the propagation characteristics of Airy beam in the Kerr media is similar to that of initial amplitude. However, it should be noted that the equilibrium effect required for a soliton state is more sensitive to the change of initial amplitude than the change of nonlinear refractive index. Interestingly, we show that, in addition to the normal breathing solitons, the anomalous bound states of Airy spatial soliton can also be formed, which are similar to the states formed in the interaction of two Airy beams in nonlinear media. This quasi-equilibrium state is formed by the interaction between a main soliton beam and the side lobes of the Airy beam due to their different propagation trajectories in the Kerr media. Moreover, it has been shown that the formed Airy solitons can propagate in a special "tree" spatial structure by adjusting the initial parameters in the interaction between the Airy beam and Kerr media.

Author Contributions: Data curation, Q.Y.; methodology, Y.-L.W.; software, L.S. All authors have read and agreed to the published version of the manuscript.

Funding: This research was funded by Anhui Provincial Natural Science Foundation, grant number 1908085QF275 and the Open Research Fund of State Key Laboratory of Pulsed Power Laser Technology, grant number SKL2019KF08 and research project of the National University of Defense Technology, grant number ZK20-41.

Acknowledgments: This work was supported by Anhui Provincial Natural Science Foundation under grant (No. 1908085QF275) and the Open Research Fund of State Key Laboratory of Pulsed Power Laser Technology under grant (No. SKL2019KF08) and research project of the National University of Defense Technology under Grant (No. ZK20-41).

Conflicts of Interest: The authors declare no conflict of interest.

\section{References}

1. Berry, M.V.; Balazs, N.L. Nonspreading wave packets. Am. J. Phys. 1979, 47, 264. [CrossRef]

2. Siviloglou, G.A.; Christodoulides, D.N. Accelerating finite energy Airy beams. Opt. Lett. 2007, 32, 979. [CrossRef] 
3. Siviloglou, G.A.; Broky, J.; Dogariu, A.; Christodoulides, D.N. Observation of accelerating Airy beams. Phys. Rev. Lett. 2007, 99, 21390111. [CrossRef]

4. Longhi, S. Airy beams from a microchip laser. Opt. Lett. 2011, 36, 716. [CrossRef] [PubMed]

5. Cheng, Z.; Zhao, S.; Chu, X.; Deng, B.; Zhang, X. Research progress of the generation methods of Airy beam. Laser Optoelectron. Prog. 2015, 52, 0081.

6. Noa, V.B.; Yossi, L.; Yigal, L.; Avraham, G.; Ady, A. Generation of electron Airy beams. Nature 2013, 494, $331-335$.

7. Khilo, N.A.; Belyi, V.N.; Kazak, N.S.; Ropot, P.I. Acoustooptic refraction- influenced generation of tunable incomplete Airy beams. J. Opt. 2014, 16, 085702. [CrossRef]

8. Ring, J.D.; Howls, C.J.; Dennis, M.R. Incomplete Airy beams: Finite energy from a sharp spectral cutoff. Opt. Lett. 2013, 38, 1639-1641. [CrossRef] [PubMed]

9. Peng, Y.; Peng, X.; Chen, B.; Zhou, M.; Chen, C.; Deng, D. Interaction of Airy-Gaussian beams in Kerr media. Opt. Commun. 2016, 359, 116-122. [CrossRef]

10. Chen, B.; Chen, C.; Peng, X.; Peng, Y.; Zhou, M.; Deng, D.; Guo, H. Evolution of the ring Airy-Gaussian beams with a spiral phase in the Kerr medium. J. Opt. 2016, 18, 1-10. [CrossRef]

11. Qian, Y.; Zhang, S. Quasi-Airy beams along tunable propagation trajectories and directions. Opt. Express 2016, 24, 9489. [CrossRef]

12. Panagiotopoulos, P.; Couairon, A.; Kolesik, M.; Papazoglou, D.G.; Moloney, J.V.; Tzortzakis, S. Nonlinear plasma-assisted collapse of ring-Airy wave packet. Phys. Rev. A 2016, 93, 0338081. [CrossRef]

13. Chen, B.; Chen, C.; Peng, X.; Peng, Y.; Zhou, M.; Deng, D. Propagation of sharply autofocused ring Airy Gaussian vortex beams. Opt. Express 2015, 23, 19288-19298. [CrossRef]

14. Wang, X.; Li, Q.; Wang, Q. Arbitrary scanning of the Airy beams using additional phase grating with cubic phase mask. Appl. Opt. 2012, 51, 6726-6731. [CrossRef] [PubMed]

15. Wang, X.; Li, Q.; Xiong, Z.; Zhang, Z.; Wang, Q. Generation and scanning of Airy beams array by combining multiphase patterns. Appl. Opt. 2013, 52, 3039-3048. [CrossRef]

16. Hu, Y.; Zhang, P.; Lou, C.; Huang, S.; Xu, J.; Chen, Z. Optimal control of the ballistic motion of Airy beams. Opt. Lett. 2010, 35, 2260-2262. [CrossRef] [PubMed]

17. Bleckmann, F.; Minovich, M.; Frohnhaus, J.; Neshev, D.N.; Linden, S. Manipulation of Airy surface Plasmon beams. Opt. Lett. 2013, 38, 1443-1445. [CrossRef]

18. Panagiotopoulos, P.; Papazoglou, D.G.; Couairon, A.; Tzortzakis, S. Sharply autofocused ring-Airy beams transforming into non-linear intense light bullets. Nat. Commun. 2013, 3622, 1-6. [CrossRef] [PubMed]

19. Abdollahpour, D.; Suntsov, S.; Papazoglou, D.G.; Tzortzakis, S. Spatiotemporal Airy light bullets in the linear and nonlinear regimes. Phys. Rev. Lett. 2010, 105, 2539011-2539014. [CrossRef]

20. Chen, R.; Yin, C.; Chu, X.; Wang, H. Effect of Kerr nonlinearity on an Airy beam. Phys. Rev. A 2010, 82, 043832. [CrossRef]

21. Kaminer, I.; Segev, M.; Christodoulides, D.N. Self-Accelerating Self-Trapped Optical Beams. Phys. Rev. Lett. 2011, 106, 213903. [CrossRef] [PubMed]

22. Lotti, A.; Faccio, D.; Couairon, A.; Papazoglou, D.G. Stationary nonlinear Airy beams. Phys. Rev. A 2011, 84, 021807. [CrossRef]

23. Zhang, Y.; Beli, M.; Wu, Z.; Zheng, H.; Lu, K.; Li, Y.; Zhang, Y. Soliton pair generation in the interactions of Airy and nonlinear accelerating beams. Opt. Lett. 2013, 38, 4585-4588. [CrossRef] [PubMed]

24. Zhang, Y.; Beli, M.R.; Zheng, H.; Chen, H.; Li, C.; Li, Y.; Zhang, Y. Interactions of Airy beams nonlinear accelerating beams and induced solitons in Kerr and saturable nonlinear media. Opt. Express 2014, 22, 7160-7171. [CrossRef] [PubMed]

25. Fattal, Y.; Rudnick, A.; Marom, D.M. Soliton shedding from Airy pulses in Kerr media. Opt. Express 2011, 19, $17298-17307$. [CrossRef] [PubMed]

26. Allayarov, M.; Tsoy, E.N. Dynamics of Airy beams in nonlinear media. Phys. Rev. A 2014, 90, 023852. [CrossRef]

27. Shi, Z.; Xue, J.; Zhu, X.; Xiang, Y.; Li, H. Interaction of Airy-Gaussian beams in photonic lattices with defects. Phys. Rev. E 2017, 95, 042209. [CrossRef]

28. Bekenstein, R.; Schley, R.; Mutzafi, M.; Rotschild, C.; Segev, M. Optical simulations of gravitational effects in the NewtonSchrödinger system. Nat. Phys. 2015, 11, 872. [CrossRef]

29. Zhan, K.; Yang, Z.; Jiao, R.; Liu, B.; Han, G.; Xu, X.; Jiao, Z. Controllable interaction of Airy beams via initial launch angle in Kerr media. Opt. Commun. 2019, 432, 49-53. [CrossRef]

30. Rudnick, A.; Marom, D.M. Airy-soliton interactions in Kerr media. Opt. Express 2011, 19, 25570. [CrossRef]

31. Olivier, V.; Manuel, S. Airy Functions and Applications to Physics 2004; Imperial College Press: London, UK, $2004 ;$ p. 55. 